\title{
T cell co-stimulator inducible co-stimulatory (ICOS) exerts potential anti-atherosclerotic roles through downregulation of vascular smooth muscle phagocytosis and proliferation
}

\author{
Zhixiong Zhong ${ }^{1,2,3}$, Qunji Zhang ${ }^{1,2,3 \#}$, Linkai Tan ${ }^{1,2,3}$, Xuemin Guo ${ }^{1,2,3}$, Caiyan Gan ${ }^{1,2,3}$ \\ ${ }^{1}$ Center for Precision Medicine, Meizhou People's Hospital, Meizhou, China; ${ }^{2}$ Guangdong Provincial Key Laboratory of Precision Medicine and \\ Clinical, Translational Research of Hakka Population, Meizhou, China; ${ }^{3}$ Guangdong Provincial Engineering and Technological Research Center for \\ Molecular Diagnostics of Cardiovascular Diseases, Meizhou, China \\ Contributions: (I) Conception and design: Z Zhong; (II) Administrative support: Z Zhong; (III) Provision of study materials or patients: Z Zhong; (IV) \\ Collection and assembly of data: Q Zhang; (V) Data analysis and interpretation: X Guo, C Gan; (VI) Manuscript writing: All authors; (VII) Final \\ approval of manuscript: All authors. \\ "These authors contributed equally to this work. \\ Correspondence to: Zhixiong Zhong. Center for Precision Medicine, Meizhou People’s Hospital, Meizhou, China. Email: zhongzhixiong@mzrmyy.com.
}

\begin{abstract}
Background: Atherosclerosis (AS) is a chronic inflammatory disease. The role of the immune system in the etiology of the disease, particularly $\mathrm{T}$ cells, has been widely studied and is well established. $\mathrm{T}$ cell activation directly regulates co-signaling molecules present in immune synapses. Targeting one or several of these cosignaling molecules can inhibit $\mathrm{T}$ cell-mediated inflammation and delay or reduce AS. In recent years, this strategy has increasingly become a research focus. As such, we explored the role and therapeutic potential of the T cell co-stimulatory molecule inducible co-stimulatory (ICOS) in AS.

Methods: We compared the expression of ICOS in early AS lesions occurring in ApoE-deficient (ApoE-KO) rats fed a fat-diet and wild type (WT) rats fed the same diet. Eight-week old ApoE-KO and WT rats [ApoE$\mathrm{KO}(0)$ and WT(0)] were fed a high-fat diet for 16 weeks [ApoE-KO(16) and WT(16)]. ICOS expression in aortic tissues was analyzed by quantitative real-time PCR, western blot, and confocal microscopy. The effect of ICOS overexpression in a transfected human $\mathrm{T}$ cell line on the phagocytosis and proliferation of cocultured human aortic smooth muscle cells (HASMCs) was studied in vitro.

Results: Compared with WT(0), ApoE-KO(0), and WT(16) rats, ICOS expression in ApoE-KO(16) rats was significantly down-regulated both at the mRNA and protein levels. In vitro experiments indicated that ICOS overexpression reduces phagocytosis and proliferation by HASMCs, and may therefore produce an anti-atherosclerotic effect.

Conclusions: The immune synaptic co-signaling molecule ICOS has an anti-atherosclerotic effect through inhibition of HASMC phagocytosis and proliferation, and can be used to delay plaque formation during the early stages of AS.
\end{abstract}

Keywords: Atherosclerosis (AS); immune system; lymphocyte; co-signaling molecules

Submitted Oct 16, 2020. Accepted for publication Dec 04, 2020.

doi: $10.21037 / \mathrm{atm}-20-7342$

View this article at: http://dx.doi.org/10.21037/atm-20-7342

\section{Introduction}

Atherosclerosis (AS) is a chronic inflammatory disease affecting the arterial walls. Identifying the molecular and cellular pathways that promote or inhibit inflammation of the arterial wall is the first step towards targeted immunotherapy for AS (1-3). Under normal conditions, low-density lipoproteins (LDL) circulate in the lumen. However, during hypertension, diabetes, and other 


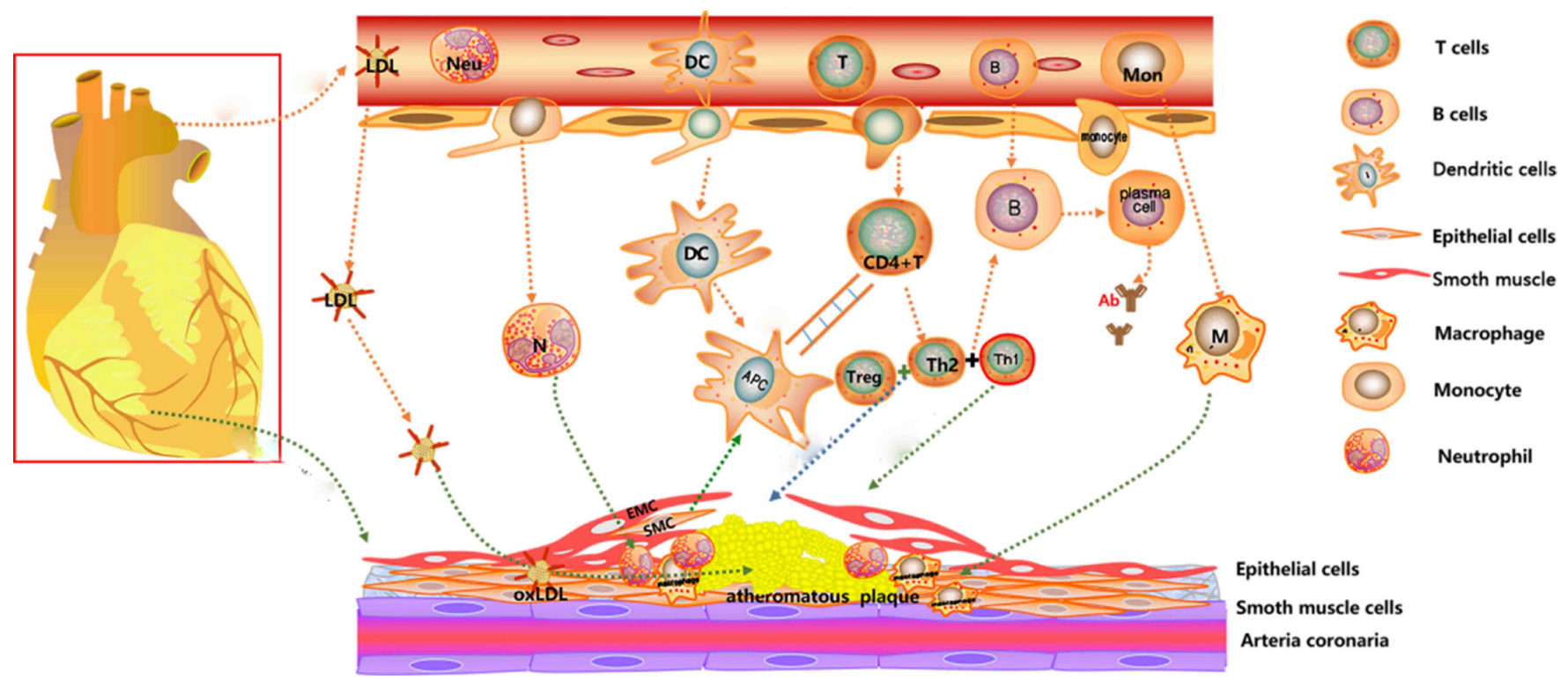

Figure 1 Involvement of immune cells during the formation of atherosclerosis. Following vascular endothelial damage caused by high-risk factors, low-density lipoprotein (LDL), normally circulating in the blood, enters the vascular endothelium and oxidizes to oxLDL. OxLDL induces endothelial cells to produce pro-inflammatory factors that attract peripheral monocytes/macrophages, dendritic cells, granulocytes, and $\mathrm{T}$ and $\mathrm{B}$ lymphocytes which infiltrate the forming plaque. Monocytes/macrophages and T lymphocytes are first activated in the endothelium and initiate innate responses. This activation leads to antigen-presenting cell maturation that, in turn, triggers $\mathrm{T}$ lymphocytes and initiates adaptive responses. Macrophages and smooth muscle cells engulf oxLDL to form foam cells, and then release oxLDL to form a lipid core following apoptosis. Smooth muscle cells migrate and proliferate to cover the lipid core and form plaques.

conditions which cause vascular endothelial damage, LDL enters the vascular endothelium and is oxidized to oxLDL. As an endogenous antigen, oxLDL stimulates endothelial cells to produce pro-inflammatory factors, causing monocytes/macrophages, dendritic cells, neutrophils, $\mathrm{T}$ and $\mathrm{B}$ lymphocytes and other inflammatory cell infiltration (4-6). Inflammation and immune cell activation are important factors for the occurrence and development of AS $(6,7)$. Monocytes and T lymphocytes are the first immune cells recruited to the endothelium. Once in the endothelium, monocytes differentiate into macrophages that produce oxygen free radicals and enzyme proteins, ingest lipoproteins through scavenger receptors, and initiate innate responses. Macrophages, endothelial cells, vascular smooth muscle cells (SMCs) and other antigen-presenting cells (APCs) then process antigens present in the plaque, and activate adaptive $\mathrm{T}$ lymphocytes $(1,8-13)$. With the occurrence of oxidation, macrophages and vascular SMCs phagocytose oxLDL to form foam cells. Upon apoptosis, foam cells release free oxLDL and form a lipid core. This attracts SMCs that proliferate in situ and form a fibrous cap covering the lipid core to form plaques (14-16). Thus, the main pathological changes and plaque formation in AS result from complex processes involving damage, adhesion, phagocytosis, and proliferation of vascular endothelial cells and SMCs, accompanied by the infiltration of large numbers of inflammatory cells such as monocytes/macrophages and T lymphocytes (Figure 1).

In recent years, the role of immune cells and molecules in the occurrence and development of AS has attracted increasing attention. A large number of studies have shown that $\mathrm{T}$ cells play an important role in AS $(8,12,14,16)$. $T$ cell activation requires two signals: firstly, an antigenspecific signal, triggered by the recognition of peptideMHC complexes (p-MHC) on the surface of APCs by $\mathrm{T}$ cell receptors (TCR); and a second co-stimulatory signal, delivered by accessory surface molecules present in the immune synapses formed at the interface of $\mathrm{T}$ cell/APC interaction $(17,18)$. Therefore, regulating cosignaling molecules on the $\mathrm{T}$ cell surface may change the intermolecular interactions between T cells and APCs, including monocytes, macrophages, endothelial cells, and 


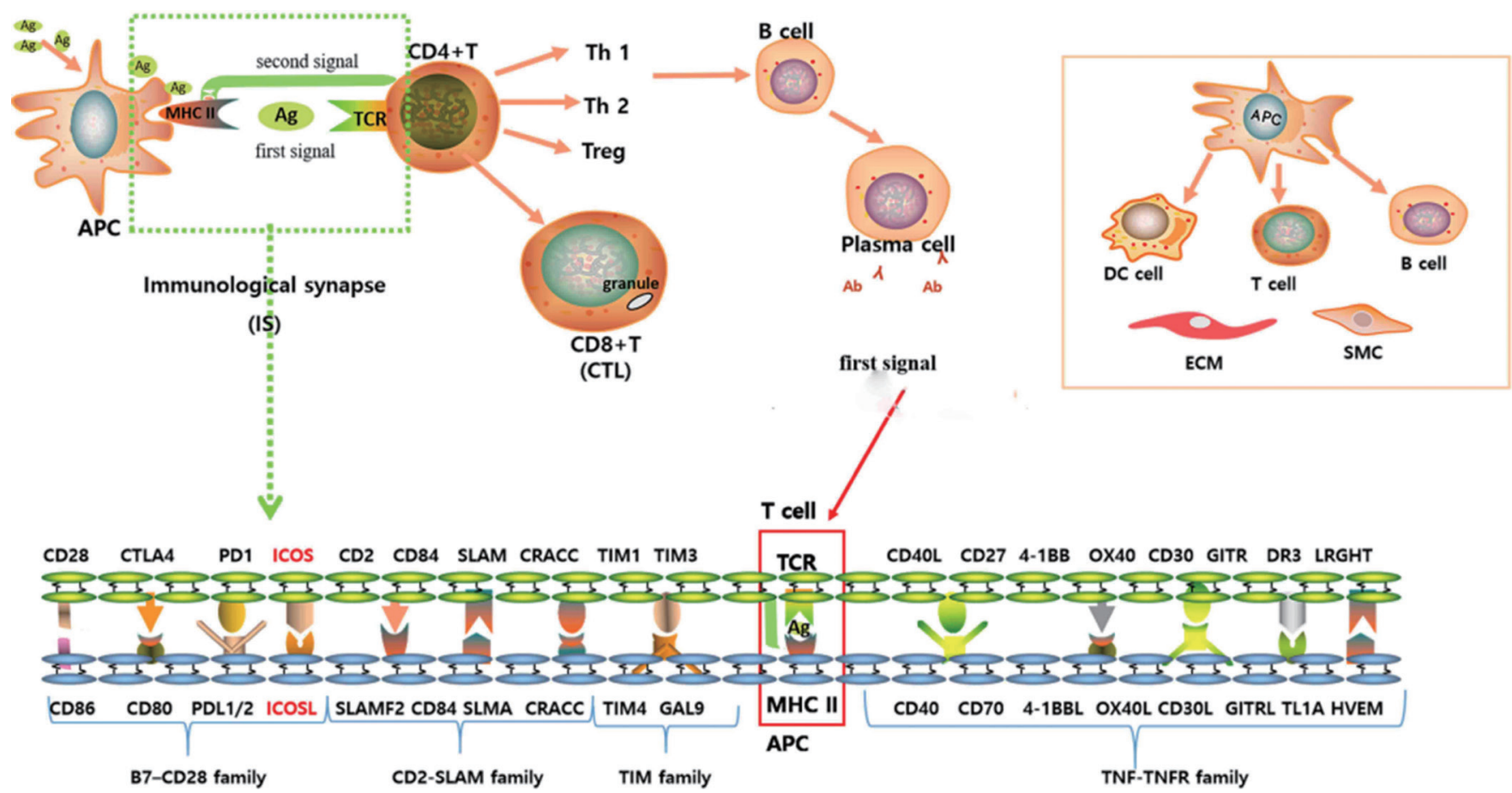

Figure $2 \mathrm{~T}$ cell activation and co-signaling molecules associated with atherosclerosis. $\mathrm{T}$ cell activation requires two signals: firstly, a signal resulting from $\mathrm{T}$ cell receptor (TCR) recognition of the cognate peptide-MHC complex (p-MHC) on the surface of antigen-presenting cells (APC); and a second signal from co-stimulatory molecules, which are potentially antagonized by co-inhibitory molecules. Co-stimulatory molecules belong to 4 families of proteins: the B7-CD28 family, TNF-TNFR family, TIM family and CD2-SLAM family.

SMCs, at the surface of the blood vessel walls. Studying the role of co-stimulatory molecules in the context of AS may therefore reveal a new perspective on atherosclerogenesis and provide potential targets for immunotherapy.

Immune synapses are special structures formed at the site of cell-cell contact during the interaction between APCs and $\mathrm{T}$ cells. The central area of the contact zone contains the TCR/p-MHC complex, surrounded by a large number of accessory molecules (Figures 1,2) (19-21). Among these accessory molecules are the co-stimulatory molecules, a class of transmembrane glycoproteins expressed by $\mathrm{T}$ cells, and their ligands on the APCs. These molecules stabilize the TCR/antigen p-MHC complexes recognition and provide auxiliary signals necessary for complete activation of the $T$ cells, thereby promoting activation, proliferation, and differentiation $(22,23)$. In the absence of this second signal, the $\mathrm{T}$ cells are not activated and become anergic. To date, a variety of co-stimulatory molecules have been identified and belong to 4 protein families: the B7-CD28 family, the tumor necrosis factor (TNF)-TNFR family, the TIM family, and the less studied family of CD2-SLAM proteins (Figure 2) (18,23-25). Each family contains multiple members, and our present study focuses on the inducible co-stimulatory (ICOS) protein belonging to the CD28 family (23,26-29). Herein, we investigated the potential anti-atherosclerosis effect of ICOS overexpression in T cells on and plaque formation through a series of in vitro and in vivo experiments. ApoE-deficient (KO) rats and cells model were used to analyze whether ICOS could inhibit AS lesions and plaque formation during the early stages of AS and then elucidate the underlying mechanism. The ApoE-KO rat model was used as a classic model of atherosclerosis.

We present the following article in accordance with the ARRIVE reporting checklist (available at http://dx.doi. org/10.21037/atm-20-7342).

\section{Methods}

\section{Animals and diet}

ApoE-deficient (KO) rats on a Sprague-Dawley (SD) background were purchased and raised at the East China Normal University animal house. Wild type (WT) rats 
were raised along with the ApoE-KO rats. Eight-weekold male WT or ApoE-KO rats were fed a Western highfat diet containing $40 \%$ of calories from fat, $43 \%$ from carbohydrates, and 17\% from proteins (D12079B, research diets) for 16 weeks, with the WT and ApoE-KO rats feeding with normal diet as the control. The experiments were approved by the Animal Ethics Committee of East China Normal University (Permit number: M20150505), in compliance with the guidelines of Ethics Committee of East China Normal University for the care and use of animals.

\section{Assessment of early AS}

The rats were divided into two groups with each group consisting of 5 animals. They were fed a western style high-fat diet for 16 weeks, and their body weight was monitored weekly. After 16 weeks, the sera were collected and tested for total cholesterol (TC), triglycerides (TG), LDL cholesterol (LDL-C), and high-density lipoprotein cholesterol (HDL-C). The entire aorta was dissected and stained with oil red O (Sigma, O0625) to assess the degree of AS. During high-fat feeding, the weight of the rats was recorded once a week to analyze their growth curves. Before the analysis, the rats were fasted for 12 hours. Blood was withdrawn from the orbital vein, and the serum was separated by centrifugation at $3,500 \mathrm{rpm}$ for $10 \mathrm{~min}$ at $4{ }^{\circ} \mathrm{C}$. The lipid content (TC, TG, LDL-C, and HDL-C) was analyzed with a AU680 automatic biochemical analyzer (Beckman Coulter, USA). The intact aorta of the rat was stripped, and the fat tissue around the blood vessel was removed with forceps as much as possible. The blood vessel was fixed for more than 24 hours, washed twice with PBS, and carefully cut longitudinally with dissecting scissors. The cut blood vessel was washed with tap water for $5 \mathrm{sec}$, immersed in $60 \%$ isopropanol for $3 \mathrm{sec}$, and stained in oil red $\mathrm{O}$ staining solution at $37{ }^{\circ} \mathrm{C}$ in the dark for $60 \mathrm{~min}$. The blood vessel was then immersed in $60 \%$ isopropanol for differentiation, until only the fat plaques in the lumen appeared orange-red or bright red, and the other parts appeared nearly colorless. The differentiation was terminated by a wash with distilled water. The excess water was absorbed on a filter paper. The aorta was then spread onto a glass slide against a black background with a scale and imaged.

\section{Real-time PCR}

Total RNA was extracted from rat aortas with the RNeasy
Fiber Tissue Mini Kit (QIAGEN, Item No. 74704), a special RNA extraction kit designed for high fiber tissues. The genomic DNA was digested and the RNA was reverse transcribed into cDNA with the PrimeScript ${ }^{\mathrm{TM}} \mathrm{RT}$ Master Mix (Takara, cat: RR036A). All real-time PCR reactions were performed with the ABI 7500 PCR detection system (Applied Biosystems; Thermo Fisher Scientific, Inc., USA) using the TB Green ${ }^{\circledR}$ Premix Ex Taq $^{\text {TM }}$ II (Takara, cat: RR820A). The following primers were used: Rat GAPDH forward, ATGACTCTACCCACGGCAAG, and reverse, ACTGTGGTCATGAGCCCTTC; rat ICOS forward, CTACTTCTCGTGCGTCTT, and reverse, GCTTCCCTTGGTCTTG. The relative expression of Icos mRNA was calculated using the $\Delta \Delta \mathrm{Ct}$ method, and statistical analysis was performed using Prism7 (GraphPad Software, Inc., USA).

\section{Western blot}

The aorta was cut into small pieces, lysed in RIPA buffer, and homogenized with a glass homogenizer. The suspension was transferred to a $1.5 \mathrm{~mL}$ microtube and centrifuged at $12,000 \mathrm{~g}$ at $4{ }^{\circ} \mathrm{C}$ for 5 minutes. The supernatant was collected and analyzed by western blot. After transfer, the membrane was blocked with 5\% BSA at room temperature for 1 hour, and incubated with the primary antibody (ICOS antibody: Biorbyt, orb314633) overnight. The membrane was washed 3 times with PBS and incubated with the secondary antibody (horse radish peroxidase-(HRP)-conjugated goat anti-rabbit IgG $(\mathrm{H}+\mathrm{L})$; Invitrogen, 65-6120) for 1 hour at room temperature. The staining was revealed by incubating the membrane with the Chemiluminescent HRP Substrate (Millipore). Protein bands were detected by a ChemiDocTM MP Imaging System (BIO-RAD).

\section{Confocal microscopy}

Rat aortas were fixed with $4 \%$ PFA, embedded in paraffin, and cut into $4 \mu \mathrm{m}$ slices. The tissue sections were deparaffinized and rinsed in water. After antigen retrieval and blocking in BSA solution, sections were incubated with the primary anti-ICOS antibody (Abcam, ab175401) then the secondary antibody (Alexa Fluor ${ }^{\circledR} 488$-conjugated polyclonal goat anti-Rabbit IgG-H\&L; Abcam, ab150077). Control slides were prepared according to the same procedure but without the primary antibody. The nuclei 
were counterstained with DAPI. Sections were mounted with a medium containing an anti-fluorescence quencher, and images were captured with a confocal microscope (LSM800, Zeiss, Oberkochen, Germany).

\section{Overexpression of ICOS in cell lines}

Lentiviral packaging technology was used to construct a Jurkat $\mathrm{T}$ cell line with stable overexpression of ICOS. The lentiviral packaging kit was purchased from Shanghai Genechem Co., Ltd. T cells were seeded in a 24-well plate at a density of $10^{5}$ cells $/ \mathrm{ml}$. The amount of virus and HiTransG P infection solution added to the culture was calculated according to the cell multiplicity of infection (MOI) and virus titer. The cells were centrifuged at $1,000 \mathrm{~g}$ for $60 \mathrm{~min}$ and cultured at $37^{\circ} \mathrm{C}$ for $12-16$ hours. Subsequently, the medium was changed and the cells were further expanded. At 72 hours after infection, $3 \mu \mathrm{g} / \mathrm{mL}$ of puromycin was added for selection. The infection rate measured by flow cytometry reached $97 \%$. The concentration of puromycin was then reduced by half for further expansion. After selection, the stable overexpressing lines were confirmed by qPCR, western blot, and flow cytometry. The following primers were used: human GAPDH forward, GAGGTTTTGAGCGTTGAGGT, and reverse, GCAGGCTGTTGTCCGTCTTA; human ICOS forward, TGCAGCCTTTGTTGTAGTCTGC, and reverse, AGGGTCACATCTGTGAGTCTAG. The following antibodies were used: recombinant anti-GAPDH antibody (Abcam, ab181602), anti-ICOS antibody (Biorbyt, orb314633), HRP-conjugated goat anti-rabbit IgG $(\mathrm{H}+\mathrm{L})$ antibody (Invitrogen, 65-6120), and PE-conjugated mouse anti-human CD278 (BD, 557802). The data were analyzed with Prism7 and FlowJo7.6.

\section{Cell cultures}

Jurkat T cells CBP60520 and human aortic smooth muscle cells HASMC60982 were used herein. T cells were cultured with RPMI 1640 medium containing 10\% FBS, whereas HASMCs were cultured with a dedicated HASMC complete medium. For co-culture, the 2 cell lines were grown in a 1:1 mix of these 2 media. For T cell/SMC coculture, HASMCs were cultured in T75 cell flasks and passaged once, then seeded into 24 -well plates $\left(10^{4} / \mathrm{mL}\right)$ and cultured for 24 hours. After adhesion, the HASMC medium was substituted by the mixed medium and supplemented with $150 \mu \mathrm{g} / \mathrm{mL}$ of oxLDL (Invitrogen, L34357) for further expansion. After 24 hours, $10^{4}$ ICOS-overexpressing $\mathrm{T}$ cells were added for 48 hours of co-culture. The ability of HASMCs to phagocytose oxLDL was assessed by oil red O staining. Mock control $\mathrm{T}$ cells were transfected with an empty lentiviral vector. For the HASMCs proliferation experiment, cells were split into 24 -well plates $\left(2 \times 10^{4} / \mathrm{mL}\right)$ containing HASMC medium to allow adherence to the wall, and then changed to mixed medium and co-cultured with ICOS-overexpressing $\mathrm{T}$ cells or mock control $\mathrm{T}$ cells $\left(2 \times 10^{4}\right)$ for 72 hours. Proliferation was assessed by CCK-8 staining.

\section{Phagocytosis assay with oil red $O$ staining}

After 48 hours of T cell/HASMC co-culture, the medium and non-adherent cells were removed. HASMCs were washed 3 times with PBS and fixed with 4\% formaldehyde for 30 minutes at $37^{\circ} \mathrm{C}$. Subsequently, the cells were stained with filtered oil red $\mathrm{O}$ solution at room temperature for 30 minutes, soaked in 60\% isopropyl alcohol for 5-10 sec, and washed 3 times with PBS. Mayer hematoxylin was added for nuclear counterstain. The cells were observed and imaged under a light microscope.

\section{Assessment of proliferation by CCK-8 staining}

After 72 hours of T cell/HASMC co-culture, non-adherent $\mathrm{T}$ cells were removed, and $500 \mathrm{~mL}$ of mixed medium plus $50 \mathrm{~mL}$ of CCK-8 solution were added to the adherent HASMCs (Dojindo, Kumamoto, Japan). Cells were incubated for 1-4 hours, and absorbance of the culture was read at $450 \mathrm{~nm}\left(\mathrm{~A}_{450}\right)$ using a microplate reader (Thermo, VarioskanTM LUX). Wells from co-cultures with mock T cells served as a reference and wells with medium only were added as blank controls. The survival rate was calculated using the formula: $\left(\mathrm{A}_{450}\right.$ of co-culture well $-\mathrm{A}_{450}$ of blank well $) /\left(\mathrm{A}_{450}\right.$ of mock control well $-\mathrm{A}_{450}$ of blank well $) \times$ $100 \%$.

\section{Statistical analysis}

All experiments were repeated at least 2 or 3 times. Statistical differences between experimental groups were assessed using a $t$-test, and $\mathrm{P}<0.05$ was considered statistically significant. Statistical analysis was performed using Prism7. 
A

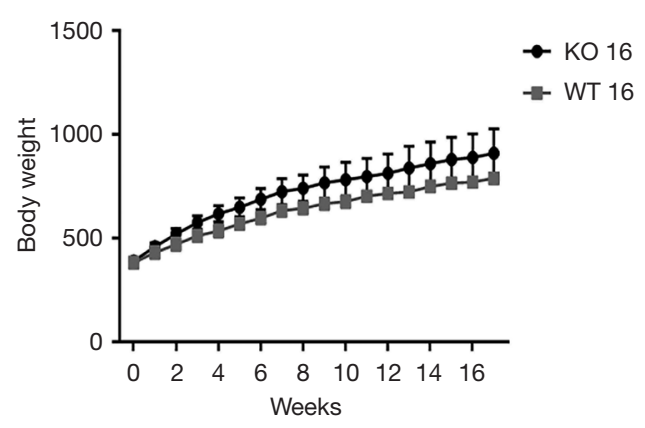

B
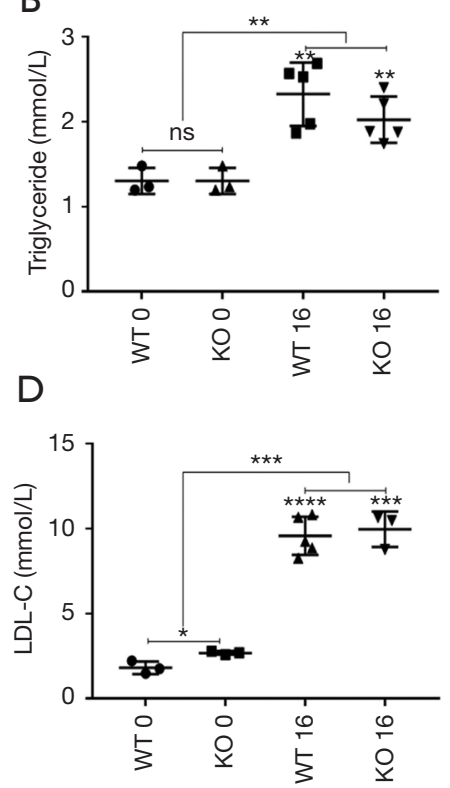

C

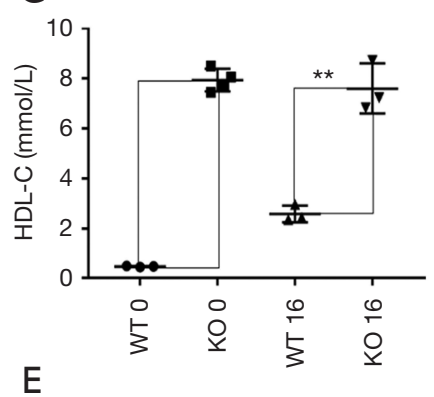

$\mathrm{E}$

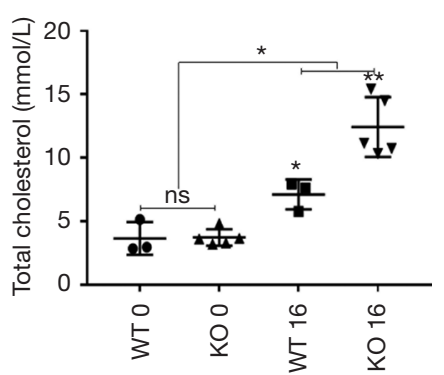

Figure 3 Monitoring of body weight and serum lipids in ApoE-knockout $(\mathrm{KO})$ and wild type (WT) rats fed a high-fat diet. Eight-week old ApoE-KO and WT rats were fed a high-fat diet for 16 weeks. (A) Body weight was monitored during the course of the high-fat diet regimen. Levels of serum lipids levels, including (B) triglycerides (TG), (C) high-density lipoprotein (HDL), (D) low-density lipoprotein (LDL), and (E) total cholesterol (TC)were compared in control 8-week old ApoE-KO and WT rats fed a normal diet (ApoE-KO(0) and WT(0), respectively), and in ApoE-KO and WT rats after 16 weeks under a high-fat diet [ApoE-KO(16) and WT(16), respectively]. *, $\mathrm{P}<0.05,{ }^{* *}, \mathrm{P}<0.01,{ }^{* * *}, \mathrm{P}<0.001,{ }^{* * * *}, \mathrm{P}<0.0001$.

\section{Results}

\section{Monitoring of weight and serum lipids in WT and ApoE- KO rats fed a bigh-fat diet}

To study the role of ICOS in early AS in vivo, we used an ApoE-KO rat model that develops AS when fed a highfat diet. Eight-week old ApoE-KO and control WT male rats were fed a high-fat diet and monitored for body weight changes. During this regimen, the body weight of the ApoE-KO rats $(\mathrm{n}=5)$ increased slightly more compared to WT rats $(\mathrm{n}=5)$, although this difference did not reach statistical significance (Figure $3 A$ ).

Next, we randomly selected 3-5 rats from each group and tested the levels of 4 blood lipids to characterize the increase of hyperlipidemia, a condition linked to cardiovascular risks, during the high-fat diet regimen. Compared to the ApoE- $\mathrm{KO}(0)$ and $\mathrm{WT}(0)$ control groups, blood TG, HDL-C, and LDL-C levels were increased in both ApoE-KO(16) and WT(16) rats fed a high-fat diet (Figure 3B,C,D). There were no significant differences between the ApoE-KO(16) and WT(16) groups for these
3 lipids. In contrast, while there was no increase in TC in $\mathrm{WT}(16)$ compared to $\mathrm{WT}(0)$ rats, the level of TC was significantly increased in $\mathrm{ApoE}-\mathrm{KO}(16)$ compared to ApoE- $\mathrm{KO}(0)$ rats (Figure $3 E$ ). Of note, the basal levels of TC, HDL-C, and LDL-C were slightly higher in ApoE$\mathrm{KO}(0)$ than in $\mathrm{WT}(0)$ rats. In summary, ApoE-KO rats that received a high-fat diet for 16 weeks had increased levels of blood TC, and in particular, increased TG, LDL-C, and HDL-C. Therefore, these rats had obvious blood lipid metabolism disorders and hyperlipidemia, both of which are inducers of early AS.

\section{Phenotypic characterization of AS by aortic oil red $O$ staining}

To assess the formation of AS in correlation with hyperlipidemia, we dissected the aortas of rats from the different experimental groups and stained them with oil red $\mathrm{O}$. No obvious plaques were found in the aortas of WT(0) and ApoE-KO(0) rats fed a normal diet (Figure 4A). After being fed a high-fat diet for 16 weeks, oil red $\mathrm{O}$ 

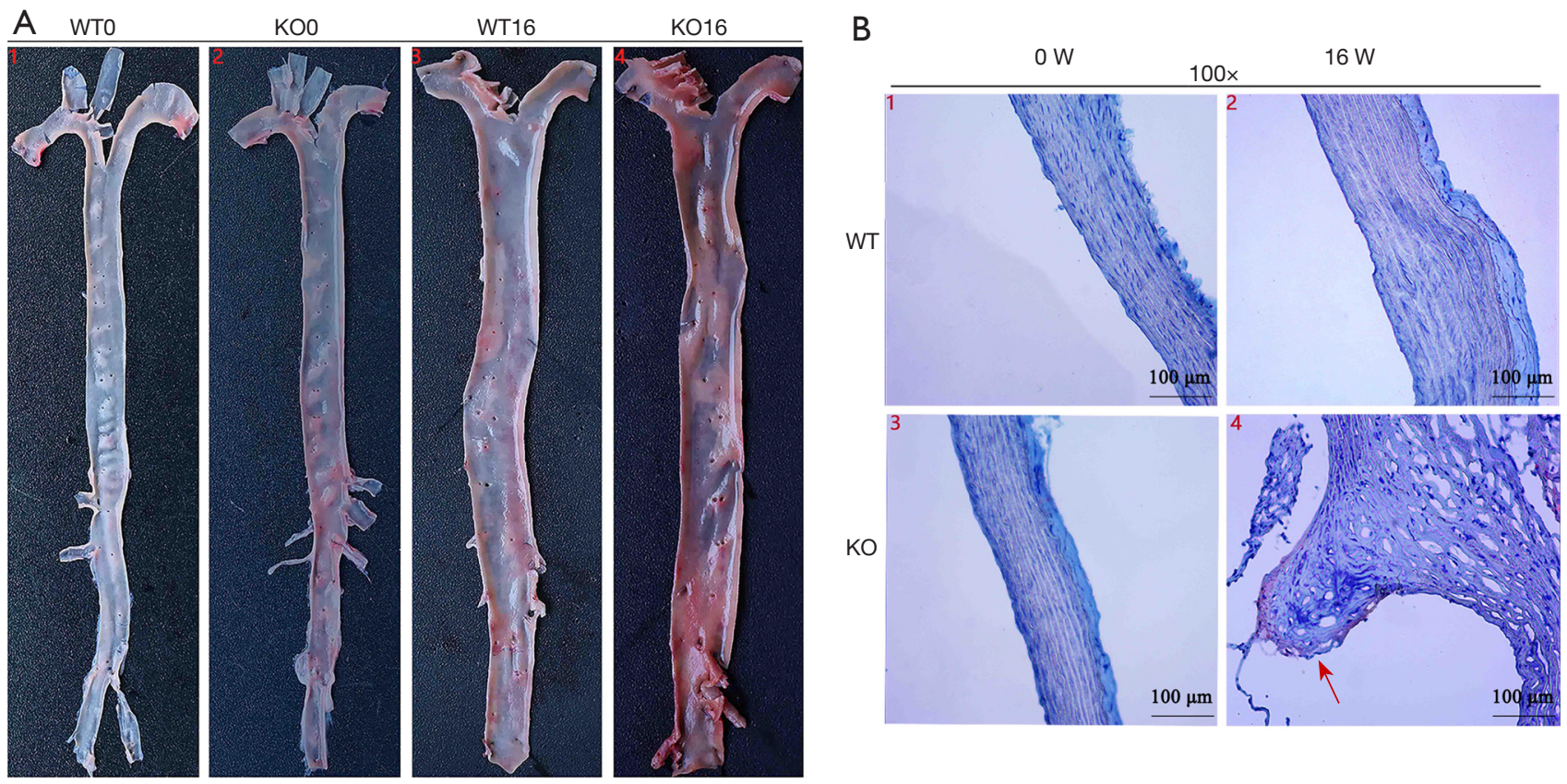

Figure 4 Phenotypic characterization of atherosclerosis by aortic oil red $\mathrm{O}$ staining. (A) Images of aortas dissected from WT(0), ApoE$\mathrm{KO}(0)$, WT(16), and ApoE-KO(16) rats and stained with oil red O to identify lipid deposition. (B) Cross section histology of aortic roots; the arrow indicates lesions in an ApoE- $\mathrm{KO}(16)$ aorta with an obvious plaque bulge and lipid deposition. Oil red $\mathrm{O}$ stained images at 100x magnifications.

staining showed that the aortas of $\mathrm{ApoE}-\mathrm{KO}(16)$ rats had obvious lipid deposition. AS was obvious at the bifurcation of the 3 vessels of the aortic arch and the lower part of the abdominal aorta. However, there was no obvious plaque bulge, which is characteristic of the early stage of AS vascular disease, or fatty streak stage (Figure $4 A$ ). In contrast, there was almost no lipid deposition in the aortic intima of WT(16) rats, and the lesions in $\operatorname{ApoE}-\mathrm{KO}(16)$ aortas were significantly increased compared with the WT(16) control group (Figure 4A). In addition, the oil red $\mathrm{O}$ staining showed that the plaque surface of the aortic root cross section from the ApoE-KO(16) rats had an obvious plaque bulge and a small amount of lipid deposition, compared with aortas from the other 3 groups (see arrows in Figure 4B). Thus, Western high-fat diets in ApoE-KO rats aggravates the formation and development of AS.

\section{Decreased expression of Icos mRNA in ApoE-KO rats}

To evaluate the potential involvement of ICOS in the development of AS, we compared the expression of Icos mRNA in the aortic tissues of WT(16) and ApoE$\mathrm{KO}(16)$ rats with that of $\mathrm{WT}(0)$ and $\mathrm{ApoE}-\mathrm{KO}(0)$ rats. We found that the expression of Icos mRNA was significantly decreased upon a high-fat diet regimen, especially in the aortic tissues of ApoE-KO(16) rats (Figure 5). Thus, the development of AS correlates with the down-regulation of Icos mRNA, suggesting that ICOS may play a protective anti-atherosclerotic role. Interestingly, even before being fed a high-fat diet, ApoE-KO(0) rats had reduced expression of Icos mRNA compared to WT(0) rats, suggesting that the down-regulation of Icos may be directly related to ApoE deletion. However, this speculation requires further investigation.

\section{Reduced expression of ICOS protein in ApoE-KO rats}

To verify if the decrease in Icos mRNA during the development of AS translated at the protein level, we compared the level of ICOS protein in aortas from $\mathrm{WT}(0)$, ApoE-KO(0), WT(16), and ApoE-KO(16) rats by western blot. Compared with $\mathrm{WT}(0)$ and $\mathrm{ApoE}-\mathrm{KO}(0)$ rats, the aortic level of ICOS was significantly reduced in WT(16) and ApoE-KO(16) rats, with a more pronounced downregulation in ApoE-KO(16) rats (Figure 6A). These results mirrored the expression levels of Icos mRNA. The reduction 


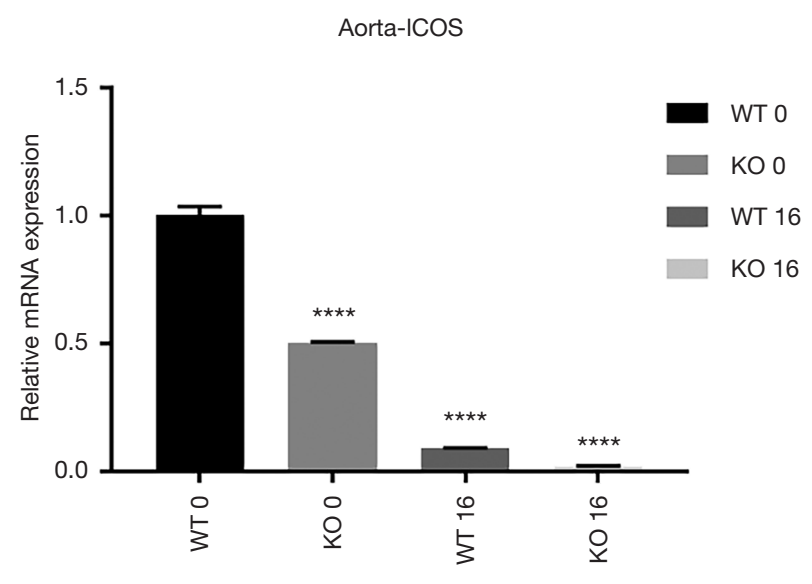

Figure 5 Relative expression of Icos mRNA in aortic tissues assessed by RT-qPCR. Relative expression of Icos mRNA in the aortic tissues of rats fed a normal [WT(0) and ApoE-KO(0)] or a high-fat diet for 16 weeks [WT(16) and ApoE-KO(16)], as assessed by RT-qPCR and normalized according to Gapdh mRNA levels. Data output and statistical analysis was completed in Prism7. ****, $\mathrm{P}<0.0001$.

of ICOS in WT(16) and ApoE-KO(16) aortas compared to $\mathrm{WT}(0)$ and $\mathrm{ApoE}-\mathrm{KO}(0)$ aortas was further confirmed by immunofluorescence imaged with confocal microscopy. As compared with WT(16) aortas, the expression of ICOS in ApoE-KO(16) aortas was significantly decreased (Figure 6B). Thus, the expression of ICOS protein decreases during AS development, implying that the reduction of ICOS molecules favors the occurrence of AS, and that ICOS may play a protective anti-atherosclerotic effect.

\section{Establishment of an in vitro system to test the effect of ICOS overexpression}

Because the downregulation of ICOS correlated with the occurrence of AS, we sought to test the effect of ICOS overexpression in a human $\mathrm{T}$ cell line (Jurkat) co-cultured with HASMCs. We constructed a lentiviral vector driving the expression of ICOS, and successfully transfected the T cells (transfection rate $>97 \%$ ). Analysis with qPCR showed that Icos mRNA levels in cultures transfected with the lentivirus containing the Icos sequence (ICOS-lentivirus group) were significantly higher than the cells transfected with the empty vector (control-lentivirus group) (Figure $7 A$ ). This overexpression was confirmed at the protein level by western blot (Figure 7B). In addition, flow cytometry directly detected the expression of ICOS protein on the cell surface, and showed that the percentage of cells expressing ICOS protein after transfection with the Icos vector was significantly higher than that in cells transfected with the mock vector (Figure 7C). These results indicated the successful establishment of a $\mathrm{T}$ cell line overexpressing ICOS protein stably.

\section{ICOS-overexpressing T cells inhibit phagocytosis and proliferation by aortic SMCs}

It is well known that phagocytosis and proliferation by SMCs are important pathological processes during plaque formation. In relation to the capacity of SMCs to phagocytose oxLDL, we tested the effect of ICOSoverexpressing $\mathrm{T}$ cells on the phagocytic ability of HASMCs. After co-culturing ICOS-overexpressing T cells or control T cells with HASMCs, the phagocytic capacity of HASMCs was assessed by oil red O staining. Compared with the control $\mathrm{T}$ cell co-culture, there were significantly fewer phagocytic vesicles with the ICOS-overexpressing $\mathrm{T}$ cell co-culture, indicating a reduced phagocytic ability of the HASMCs (Figure 8A). In the same way, we measured the effect of ICOS-overexpressing T cells on HASMC proliferation using the CCK- 8 assay. Similar to the effects on phagocytosis, the proliferation of HASMCs co-cultured with ICOS-overexpressing T cells was reduced compared to the proliferation of HASMCs co-cultured with control T cells (Figure 8B). These results suggest that ICOS may exert an anti-atherosclerotic effect by limiting the phagocytosis and proliferation by SMCs during plaque formation.

\section{Discussion}

With the great success of immunotherapy in the field of cancer, the role of the immune system in disease development and progression is receiving increasing attention (30-33). In recent years, the importance of $T$ cells in AS has been extensively studied and confirmed. Different $T$ cell subsets have different pro- or antiinflammatory functions. Th1 cells mainly promote inflammatory responses, which sometimes have damaging effects, particularly in the context of AS. The implications of Th2 cells are more ambiguous, as they have been involved in AS resistance, but at times have also shown a slight atherosclerogenic effect. By contrast, regulatory $\mathrm{T}$ cells reduce the formation of AS and play a protective role (34-39). The co-stimulatory molecules involved in immune synapses between T cells and APCs can regulate 

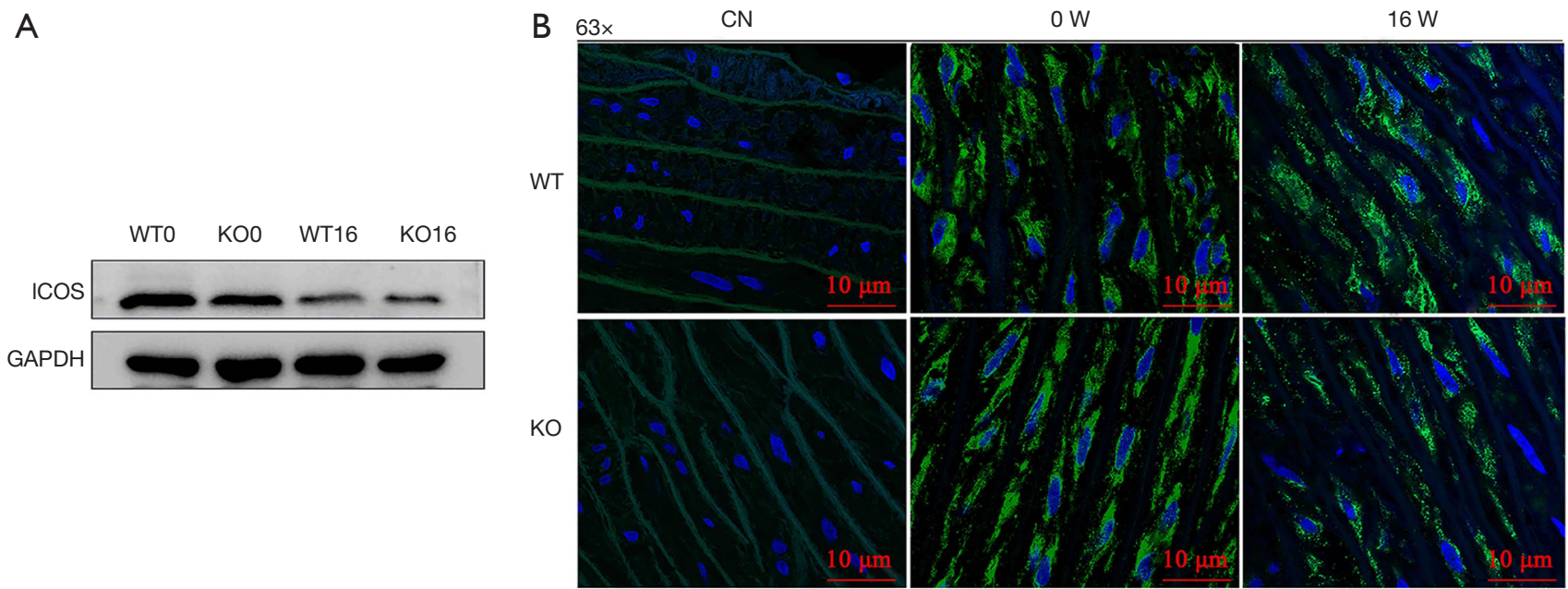

Figure 6 Comparison of ICOS protein levels in aortic tissues from healthy and atherosclerotic rats. (A) Protein extracts were prepared from the aortas of rats fed a normal [WT(0) and ApoE-KO(0)] or a high-fat diet for 16 weeks [WT(16) and ApoE-KO(16)]. ICOS protein level was quantified by western blot. (B) Fluorescence confocal microscopy images showing ICOS protein (green) in aortas of WT(0), ApoE$\mathrm{KO}(0)$, WT(16), and ApoE-KO(16) rats; nuclei were counterstained with DAPI. ICOS was revealed with a primary rabbit anti-rat ICOS antibody and a secondary Alexa488-conjugated goat anti-rabbit antibody, and control slides (CN) were stained without the primary antiICOS antibody. Fluorescence confocal microscopy images at $63 \times$ magnifications.

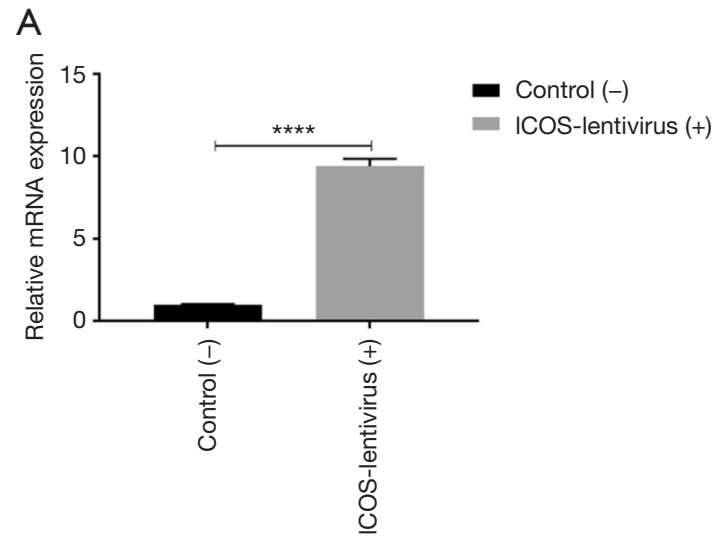

B
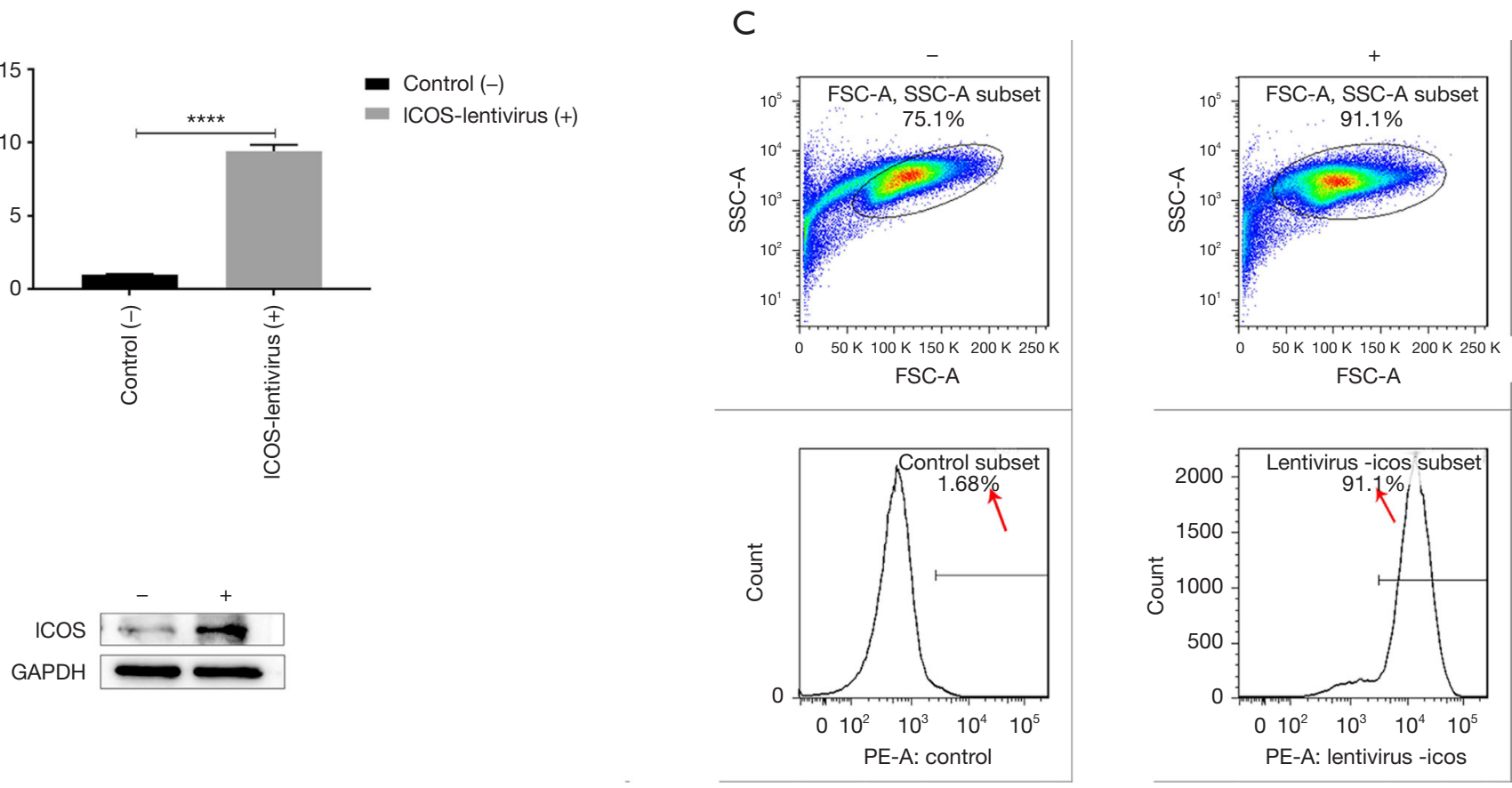

Figure 7 Establishment of a stable ICOS-overexpressing human Jurkat T cell line. (A) qPCR analysis showing Icos mRNA levels in Jurkat cells transfected with an ICOS expression vector [ICOS-lentivirus(+)], or an empty control vector [Control(-)]. (B) Western blot and (C) flow cytometry results showing ICOS protein in ICOS-lentivirus(+) and control vector [Control(-)] $\mathrm{T}$ cells. ${ }^{* * * *}, \mathrm{P}<0.0001$. 

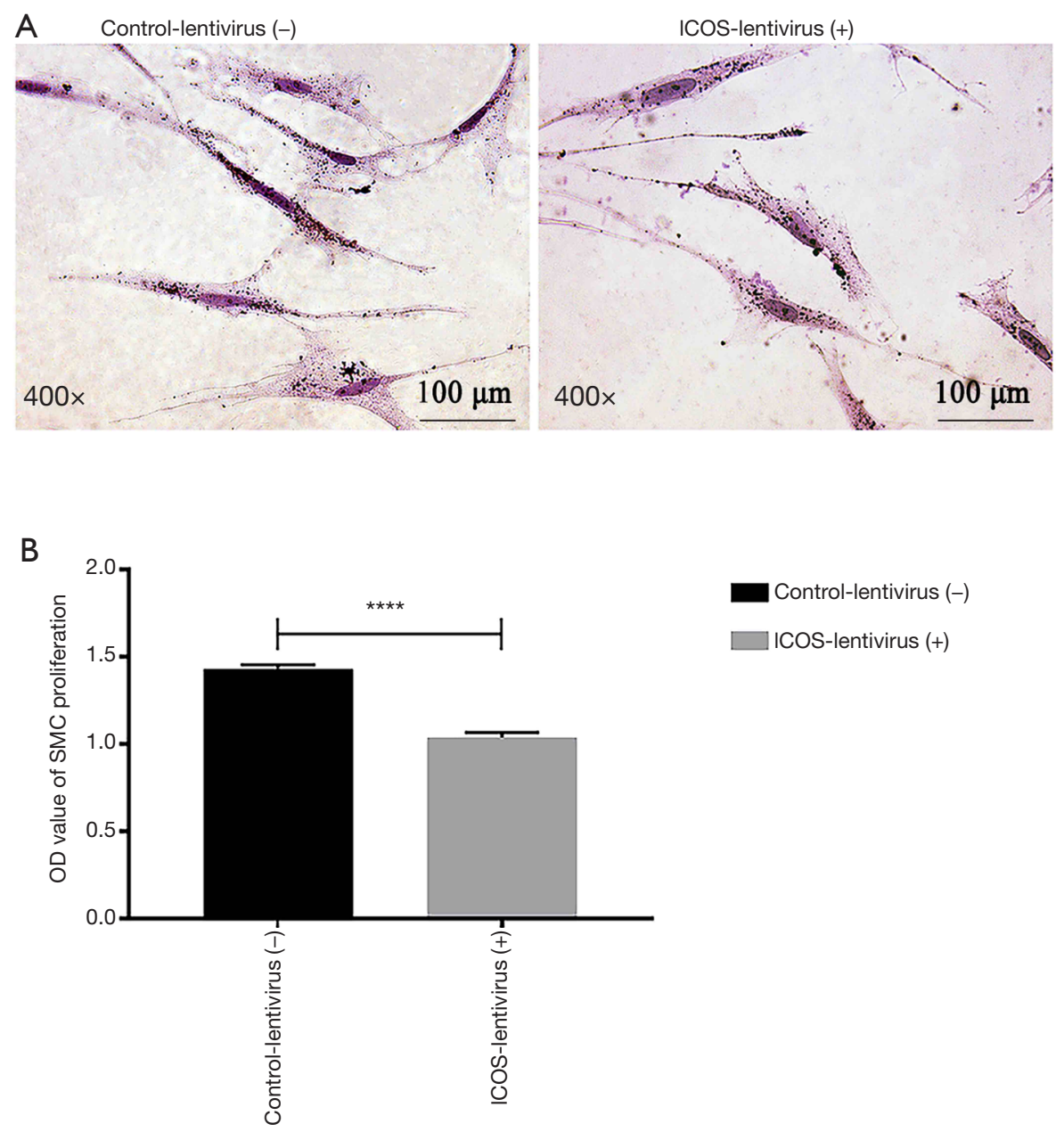

Figure 8 Inhibition of phagocytosis and proliferation in HASMCs mediated by ICOS-overexpression of T cells. (A) HASMCs were stained with oil red $\mathrm{O}$ after co-culture with $\mathrm{T}$ cells overexpressing ICOS [ICOS-lentivirus(+)] or control T cells transfected with empty vector [Control(-)]. Oil red O stained images at 400× magnifications. (B) Results summary of the CCK-8 assay performed on HASMCs after coculture with T cells overexpressing ICOS [ICOS-lentivirus(+)] or control T cells transfected with an empty vector [Control(-)] to measure cell proliferation. The statistical analysis was conducted in Prism7. ****, $\mathrm{P}<0.0001$. HASMCs, human aortic smooth cells.

the function and differentiation of $\mathrm{T}$ cells, and affect the development of AS in the blood vessel walls. A variety of co-signal molecules related to AS have been discovered, including co-stimulatory and co-inhibitory molecules $(18,23)$. In this study, we investigated a new way to influence the occurrence and development of AS by modulating the expression of one of these co-stimulatory molecules-the ICOS protein.

We found that the expression of ICOS in the aortic tissues of ApoE-KO rats was down-regulated during the formation of AS plaques, indicating that the lack of ICOS increased AS burden. Therefore, we hypothesized that
ICOS may be a protective molecule with anti-atherosclerotic effects, and we set out to elucidate through which biological mechanisms it may operate using in vitro experiments. First, lentivirus packaging technology was successfully used to construct a $\mathrm{T}$ cell line overexpressing ICOS. According to immunological processes involved in AS, we established a co-culture assay to test the effect of ICOS overexpression in $\mathrm{T}$ cells on HASMC phagocytic capacity and proliferation. As a result of overexpressing ICOS in T cells, the ability of HASMCs to phagocytose oxLDL was weakened, indicating a potential anti- atherosclerotic effect of ICOS. Overexpression of ICOS also inhibited the proliferation 
of HASMCs. This experiment in vitro further supports the hypothesis of a protective role of ICOS as suggested by the data obtained in the ApoE-KO rat model of AS. The finding that ICOS may have an anti-atherosclerotic effect by reducing the phagocytosis capacity and proliferation of vascular SMCs provides a new biological function for this protein, suggesting a possibility of being a potential drug candidate for AS treatment. However, direct evidence of a protective role of ICOS against AS should be further verified by studying the development of AS in ApoE/ICOSdouble knockout mice.

Compared with targeting inhibitory molecules to reduce $\mathrm{T}$ cell activation, blocking stimulatory molecules to reduce $\mathrm{T}$ cell activity in AS seems a safer strategy, with higher clinical prospects. However, in AS treatment, the balance between effector and regulatory $T$ cell responses is an important factor to consider (37,40-44). Blocking stimulatory molecules could damage the differentiation and function of both effector and regulatory $\mathrm{T}$ cells, thus representing a double-edged sword. In our study, although ICOS is a $\mathrm{T}$ cell co-stimulatory molecule, its reduction correlated with increased AS. This finding suggests that a lack of ICOS may affect more regulatory $T$ cell functions than impair damaging effector $\mathrm{T}$ cells. Therefore, it is possible that the number and function of regulatory $T$ cells is enhanced by the up-regulation of ICOS, which may exert an anti-atherosclerotic effect by counterbalancing effector T cells.

In conclusion, our results suggest that ICOS may play an anti-atherosclerotic role in the early stage of AS development by inhibiting the phagocytosis capacity and proliferation of SMCs. Targeting ICOS to regulate $\mathrm{T}$ cell activation may affect plaque formation and delay or reverse the occurrence and development of AS. However, the role of ICOS in AS development and the underlying mechanism need further investigation.

\section{Acknowledgments}

The author would like to thank all colleagues from the Center for Precision Medicine, Meizhou People's Hospital (Huangtang Hospital), Meizhou Academy of Medical Sciences, and Meizhou Hospital Affiliated to Sun Yat-sen University for their helpful comments on the manuscript.

Funding: This study was supported by grants from the Natural Science Foundation of Guangdong Province (2020A15150101430), the Guangdong Provincial Key Laboratory of Precision Medicine and Clinical Translation
Research of Hakka Population (2018B030322003), the Science and Technology Program of Meizhou (2019B0202001), and the Key Scientific and Technological Project of Meizhou People's Hospital (PY-A2019003).

\section{Footnote}

Reporting Checklist: The authors have completed the ARRIVE reporting checklist. Available at http://dx.doi. org/10.21037/atm-20-7342

Data Sharing Statement: Available at http://dx.doi. org/10.21037/atm-20-7342

Conflicts of Interest: All authors have completed the ICMJE uniform disclosure form (available at http://dx.doi. org/10.21037/atm-20-7342). The authors have no conflicts of interest to declare.

Ethical Statement: The authors are accountable for all aspects of the work in ensuring that questions related to the accuracy or integrity of any part of the work are appropriately investigated and resolved. The experiments were approved by the Animal Ethics Committee of East China Normal University (Permit number: M20150505), in compliance with the guidelines of Ethics Committee of East China Normal University for the care and use of animals.

Open Access Statement: This is an Open Access article distributed in accordance with the Creative Commons Attribution-NonCommercial-NoDerivs 4.0 International License (CC BY-NC-ND 4.0), which permits the noncommercial replication and distribution of the article with the strict proviso that no changes or edits are made and the original work is properly cited (including links to both the formal publication through the relevant DOI and the license). See: https://creativecommons.org/licenses/by-nc-nd/4.0/.

\section{References}

1. Gisterå A, Hansson GK. The immunology of atherosclerosis. Nat Rev Nephrol 2017;13:368-80.

2. Koltsova EK, Garcia Z, Chodaczek G, et al. Dynamic T cell-APC interactions sustain chronic inflammation in atherosclerosis. J Clin Invest 2012;122:3114-26.

3. Peng LP, Cao Y, Zhao SL, et al. Memory T cells delay the progression of atherosclerosis via AMPK signaling pathway. Ann Transl Med 2019;7:782. 
4. Chen X, Lin J, Hu T, et al. Galectin-3 exacerbates ox-LDL-mediated endothelial injury by inducing inflammation via integrin $\beta 1$-RhoA-JNK signaling activation. J Cell Physiol 2019;234:10990-1000.

5. He D, Xu L, Wu Y, et al. Rac3, but not Rac1, promotes oxLDL induced endothelial dysfunction by downregulating autophagy. J Cell Physiol 2020;235:1531-42.

6. Lin F, Pei L, Zhang Q, et al. Ox-LDL induces endothelial cell apoptosis and macrophage migration by regulating caveolin-1 phosphorylation. J Cell Physiol 2018;233:6683-92.

7. Gimbrone MA Jr, García-Cardeña G. Endothelial Cell Dysfunction and the Pathobiology of Atherosclerosis. Circ Res 2016;118:620-36.

8. Abdolmaleki F, Gheibi Hayat SM, Bianconi V, et al. Atherosclerosis and immunity: A perspective. Trends Cardiovasc Med 2019;29:363-71.

9. Ait-Oufella H, Sage AP, Mallat Z, et al. Adaptive (T and $\mathrm{B}$ cells) immunity and control by dendritic cells in atherosclerosis. Circ Res 2014;114:1640-60.

10. Herrero-Fernandez B, Gomez-Bris R, Somovilla-Crespo B, et al. Immunobiology of Atherosclerosis: A Complex Net of Interactions. Int J Mol Sci 2019;20:5293.

11. Lacy M, Atzler D, Liu R, et al. Interactions between dyslipidemia and the immune system and their relevance as putative therapeutic targets in atherosclerosis. Pharmacol Ther 2019;193:50-62.

12. Tabas I, Lichtman AH. Monocyte-Macrophages and T Cells in Atherosclerosis. Immunity 2017;47:621-34.

13. Taleb S. Inflammation in atherosclerosis. Arch Cardiovasc Dis 2016;109:708-15.

14. McLaren JE, Michael DR, Ashlin TG, et al. Cytokines, macrophage lipid metabolism and foam cells: implications for cardiovascular disease therapy. Prog Lipid Res 2011;50:331-47.

15. Witztum JL, Lichtman AH. The influence of innate and adaptive immune responses on atherosclerosis. Annu Rev Pathol 2014;9:73-102.

16. Yuan Y, Li P, Ye J. Lipid homeostasis and the formation of macrophage-derived foam cells in atherosclerosis. Protein Cell 2012;3:173-81.

17. Azuma M. Co-signal Molecules in T-Cell Activation : Historical Overview and Perspective. Adv Exp Med Biol 2019;1189:3-23.

18. Simons KH, de Jong A, Jukema JW, et al. T cell costimulation and co-inhibition in cardiovascular disease: a double-edged sword. Nat Rev Cardiol 2019;16:325-43.

19. Dustin ML, Choudhuri K. Signaling and Polarized
Communication Across the T Cell Immunological Synapse. Annu Rev Cell Dev Biol 2016;32:303-25.

20. Fooksman DR, Vardhana S, Vasiliver-Shamis G, et al. Functional anatomy of $T$ cell activation and synapse formation. Annu Rev Immunol 2010;28:79-105.

21. Reichardt P, Dornbach B, Gunzer M. The molecular makeup and function of regulatory and effector synapses. Immunol Rev 2007;218:165-77.

22. Ford ML. T Cell Cosignaling Molecules in Transplantation. Immunity 2016;44:1020-33.

23. Gotsman I, Sharpe AH, Lichtman AH. T-cell costimulation and coinhibition in atherosclerosis. Circ Res 2008;103:1220-31.

24. Rothstein DM, Sayegh MH. T-cell costimulatory pathways in allograft rejection and tolerance. Immunol Rev 2003;196:85-108.

25. Smith-Garvin JE, Koretzky GA, Jordan MS. T cell activation. Annu Rev Immunol 2009;27:591-619.

26. Carreno BM, Collins M. The B7 family of ligands and its receptors: new pathways for costimulation and inhibition of immune responses. Annu Rev Immunol 2002;20:29-53.

27. Chen LJNrI. Co-inhibitory molecules of the B7-CD28 family in the control of T-cell immunity. 2004;4:336-47.

28. Sharpe AH, Freeman GJ. The B7-CD28 superfamily. Nat Rev Immunol 2002;2:116-26.

29. Chen L. Co-inhibitory molecules of the B7-CD28 family in the control of T-cell immunity. Nat Rev Immunol 2004;4:336-47.

30. Constantinidou A, Alifieris C, Trafalis DT. Targeting Programmed Cell Death -1 (PD-1) and Ligand (PD-L1): A new era in cancer active immunotherapy. Pharmacol Ther 2019;194:84-106.

31. Evans M, O'Sullivan B, Smith M, et al. Predictive markers for anti-PD-1/PD-L1 therapy in non-small cell lung cancer-where are we? Transl Lung Cancer Res 2018;7:682-90.

32. Szeto GL, Finley SD. Integrative Approaches to Cancer Immunotherapy. Trends Cancer 2019;5:400-10.

33. Sugie T. Immunotherapy for metastatic breast cancer. Chin Clin Oncol 2018;7:28.

34. Daugherty A, Rateri DL. T lymphocytes in atherosclerosis: the yin-yang of Th1 and Th2 influence on lesion formation. Circ Res 2002;90:1039-40.

35. Ley K. Role of the adaptive immune system in atherosclerosis. Biochem Soc Trans 2020;48:2273-81.

36. Li J, McArdle S, Gholami A, et al. CCR5+T-bet+FoxP3+ Effector CD4 T Cells Drive Atherosclerosis. Circ Res 2016;118:1540-52. 
37. Saigusa R, Winkels H, Ley K. T cell subsets and functions in atherosclerosis. Nat Rev Cardiol 2020;17:387-401.

38. Yang Y, Liu Y, Chen X, et al. 5-Aminolevulinic AcidMediated Sonodynamic Therapy Alleviates Atherosclerosis via Enhancing Efferocytosis and Facilitating a Shift in the Th1/Th2 Balance Toward Th2 Polarization. Cell Physiol Biochem 2018;47:83-96.

39. Zhou X, Paulsson G, Stemme S, et al.

Hypercholesterolemia is associated with a T helper (Th) $1 /$ Th2 switch of the autoimmune response in atherosclerotic apo E-knockout mice. J Clin Invest 1998;101:1717-25.

40. Foks AC, Frodermann V, ter Borg M, et al. Differential effects of regulatory $\mathrm{T}$ cells on the initiation and regression of atherosclerosis. Atherosclerosis 2011;218:53-60.

41. Gao S, Zhang W, Zhao Q, et al. Curcumin ameliorates

Cite this article as: Zhong Z, Zhang Q, Tan L, Guo X, Gan C. $\mathrm{T}$ cell co-stimulator inducible co-stimulatory (ICOS) exerts potential anti-atherosclerotic roles through downregulation of vascular smooth muscle phagocytosis and proliferation. Ann Transl Med 2020;8(23):1597. doi: 10.21037/atm-20-7342 atherosclerosis in apolipoprotein $\mathrm{E}$ deficient asthmatic mice by regulating the balance of Th2/Treg cells. Phytomedicine 2019;52:129-35.

42. Huang Y, Hu H, Liu L, et al. Interleukin-12p35 Deficiency Reverses the Th1/Th2 Imbalance, Aggravates the Th17/ Treg Imbalance, and Ameliorates Atherosclerosis in ApoE/- Mice. Mediators Inflamm 2019;2019:3152040.

43. Li N. CD4+ T cells in atherosclerosis: regulation by platelets. Thromb Haemost 2013;109:980-90.

44. Talepoor AG, Fouladseresht H, Khosropanah S, et al. Immune-Inflammation in Atherosclerosis: A New Twist in an Old Tale. Endocr Metab Immune Disord Drug Targets 2020;20:525-45.

(English Language Editor: C. Betlazar-Maseh) 